\title{
CRYPTOCURRENCY: A MINE OF CONTROVERSIES
}

Graziela Molling https://orcid.org/0000-0003-1166-6134

Amarolinda Klein https://orcid.org/0000-0001-8585-9057

Norberto Hoppen https://orcid.org/0000-0003-1827-8695

Rafael Dalla Rosa https://orcid.org/0000-0002-4744-3357

\section{Universidade do Vale do Rio dos Sinos, São Leopoldo, RS, Brazil}

\begin{abstract}
Cryptocurrency is attracting the attention of many disciplines. Based on a systematic literature review, the state of art of academic research on cryptocurrency was investigated, demonstrating its complexity and the lack of consensus about several issues, as its definition, its operation without a financial institution, the impacts on economy and its future developments. Considering these issues, Actor-Network Theory was selected as a theory that can provide methods, such as controversy mapping, to understand this complex subject. The article analyzes this scenario, presenting a set of research topics that can be considered to study controversies related to cryptocurrency.
\end{abstract}

Keywords: Cryptocurrency, Actor-Network Theory, Controversies, Controversy mapping.

Manuscript first received: 2018-11-30. Manuscript accepted: 2020-03-01

Address for correspondence:

Graziela Molling. Universidade do Vale do Rio dos Sinos, São Leopoldo, RS, Brazil. E-mail: gmolling@edu.unisinos.br Amarolinda Klein. Universidade do Vale do Rio dos Sinos, São Leopoldo, RS, Brazil. E-mail: aczanela@unisinos.br Norberto Hoppen. Universidade do Vale do Rio dos Sinos, São Leopoldo, RS, Brazil. E-mail: norbertoh@unisinos.br Rafael Dalla Rosa. Universidade do Vale do Rio dos Sinos, São Leopoldo, RS, Brazil. E-mail: rafadalla@edu.unisinos.br 


\section{INTRODUCTION}

Cryptocurrencies such as Bitcoin, Ethereum or Litecoin have been attracting the attention of information technology professionals, economists, investors, banks, government, and even the police. This technological novelty has increased over the last years due to its innovative features, simplicity, transparency, high market price and popularity (Moore, 2013; Urquhart, 2016). Cryptocurrencies challenge the current financial systems and conventional forms of currency (Bjerg, 2016, p.53). The underlying purpose behind the cryptocurrency movement is related to the decentralization of power; they are not controlled by a central bank or government (Bariviera, Zunino, \& Rosso, 2018; Rahman, 2018). Due to this, cryptocurrencies are igniting intense discussions and controversies (Li \& Wang, 2017).

The research about cryptocurrency has increased over the years in several disciplines such as Business, Economy, Technology, Law, Philosophy, and Criminology, demonstrating the relevance of this topic (Tschorsch \& Scheuermann, 2016; Li \& Wang, 2017). These areas focus on understanding this innovation, proposing its definition, studying its benefits, risks, and impacts, its behavior especially in market and economy - and its usefulness. Attempting to contribute with this debate, we analyze the state of art of the research on cryptocurrency identifying key topics to be studied considering the method of controversy mapping (Venturini, 2010b) based on the Actor-Network Theory (ANT).

This article aims to answer the questions: a) what are the main topics presented in the literature on cryptocurrencies? b) what are the main discussions and controversies on each topic presented in the literature?

Considering cryptocurrency is a hot topic in the academic literature and in the nonacademic world, many questions have been raised as, for instance, if it will be considered or replace the currencies that we know, if is legal or not to use it or if is a good option to invest due its market price, among other several questions. However, no previous study has been found so far presenting the state of art of the literature on cryptocurrencies neither presenting the discussions and controversies about this issue. So, this study contributes to the literature about cryptocurrency, given an overview of the state of art and presenting new research opportunities that may explore the discussions and controversies around this subject.

This article is organized into four sections. In section 2 we present a literature review on cryptocurrency and the method used in this study; in section 3 we show the theoretical background ANT, and in section four, we analyze key controversies on cryptocurrency developing questions for future research. In the last section, we make concluding remarks.

\section{CRYPTOCURRENCY}

The definition of cryptocurrency is still under discussion. There is no agreement whether it can be considered money (or not) and researchers are trying to fit it in the theories of money (Šurda, 2014; Bjerg, 2016). However, there is a consensus that cryptocurrencies can be considered as a digital medium of exchange and a decentralized payment system based on cryptography (Luther \& White, 2014; Šurda, 2014; Osterrieder \& Lorenz, 2017). 
The interest on cryptocurrencies has started in 2008, with Satoshi Nakamoto's (whose real identity is still unknown) publication about a system for electronic transactions without relying on a trusted third party such as financial institutions (Nakamoto, 2008; Tschorsch \& Scheuermann, 2016). In 2009, the proposed system was released as an open-source software and Bitcoin, the first decentralized cryptocurrency and the most famous one, emerged (Maurer, Nelms, \& Swartz, 2013; Osterrieder \& Lorenz, 2017).

According to Tschorsch and Scheuermann (2016), the idea of cryptocurrency is not new. However, previous attempts (like B-Money) could not successfully address issues such as the double spending problem (when a user attempts to transfer the same coin more than once) or eliminate the need of a central bank authority (Barber, Boyen, Shi, \& Uzun, 2012; Li \& Wang, 2017). Nakamoto (2008) found the solution for these issues in the blockchain technology. The blockchain is a public distributed ledger (computer file) that saves the information of all cryptocurrencies' transactions and is stored on thousands of computers spread across the world, connected to the cryptocurrency network (Bjerg, 2016; Osterrieder \& Lorenz, 2017). A summary explaining how cryptocurrency works is presented in Figure 1.

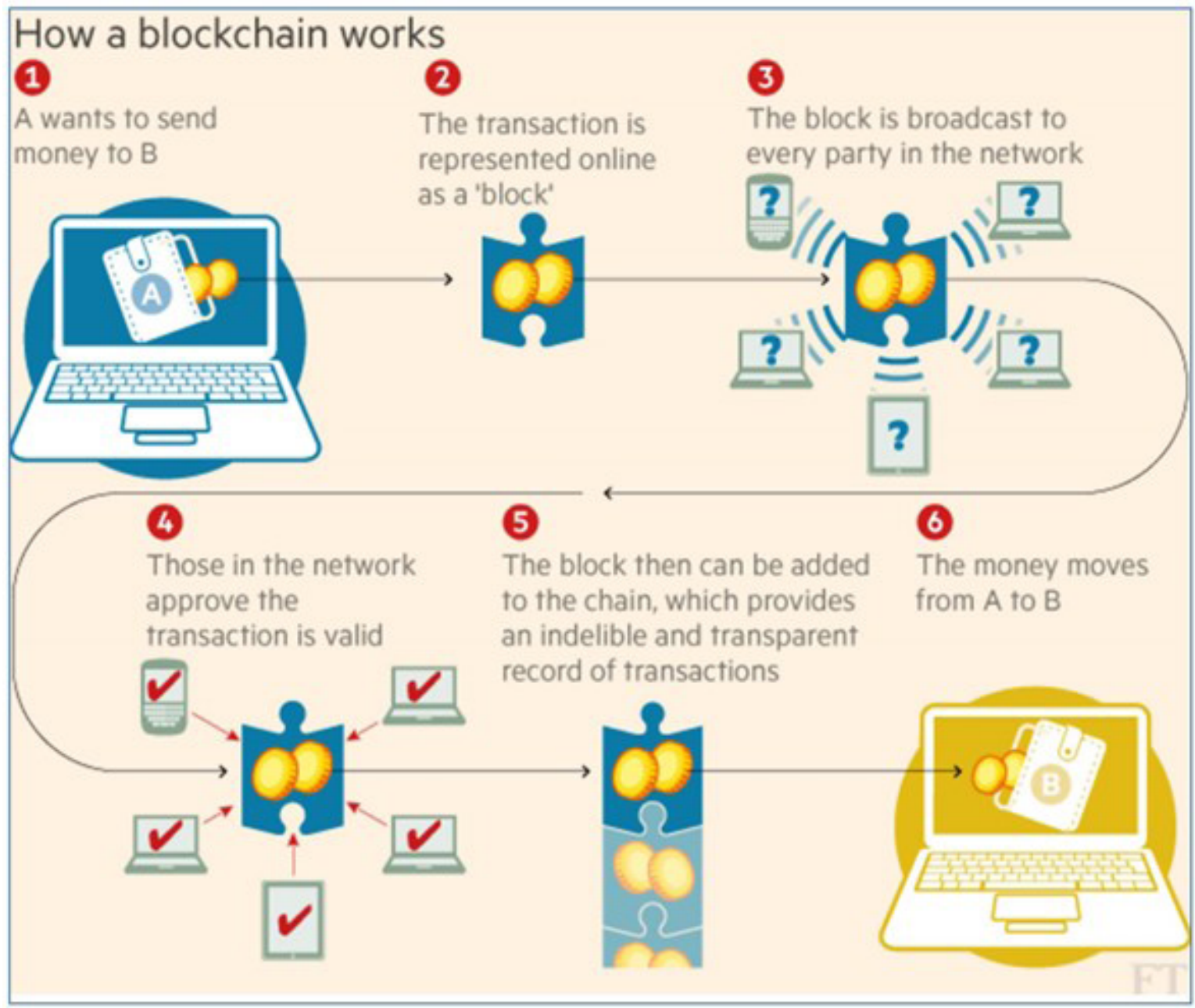

Figure 1. How cryptocurrency works Source: World Economic Forum (2016) 
Basically, the transaction is sent and validated by several computers in the cryptocurrency network. This process of validation is called "mining" and is the kernel of this technology, ensuring reliability and security, avoiding malicious attacks (Li \& Wang, 2017; Zimmer, 2017). This mining process works because several computers (named miners) connected around the world, do this process (Aste, Tasca, \& Di Matteo, 2017; Li \& Wang, 2017). However, as highlighted by Reid and Harrigan $(2013$, p. 202), the mining process "is designed to require considerable computational effort" (electricity and CPU time). Cryptocurrency schemes provide an incentive (a fee) to encourage miners to spend electricity and CPU resources in counterpart. According to Zimmer (2017, p. 308), the mining process can be compared as extracting gold, and the expenditure of resources is rewarded with cryptocurrencies "extracted from the digital bedrock".

Considering this system, instead of a centralized authority that validates the transactions, the validation is distributed (decentralized) along the cryptocurrency network, executed by the "miners". Also, the blockchain technology ensures the anonymity of the cryptocurrency owners and their transactions through the cryptography system. In the absence of this central authority, cryptocurrencies are free from government regulation, taxation, and fees from financial institutions (Kim, 2015). Nevertheless, an incentive is necessary to keep the miners working and therefore ensure the reliability and security of the payment system (Kim, 2015). In summary, a cryptocurrency system is based on the blockchain, in the public ledger that records all transactions and it is shared among the users, counting on an incentive's structure, that encourages "miners" to keep the system flowing (Redshaw, 2017).

\section{METHOD}

A systematic literature review is an essential work for the dissemination of knowledge, supporting the understanding of concepts and the construction of theories, as well as the dissemination of new research areas. Thus, through a systematic review of the literature on cryptocurrencies, we seek to carry out a consistent survey of what is considered relevant about this topic in the current research (Webster \& Watson, 2002).

This literature review follows the steps summarized in Figure 2 based on Sampaio and Mancini (2007). Based on the research questions defined in this article, a search in the Web Of Science database was performed in October 16th, 2018, limited to "Articles" as the document type. The words used to find the articles by topic (title, abstract or keywords) were: "crypto-currency", "crypto-currencies", "cryptocurrency", “cryptocurrencies”, "cryptocurrency", “cryptocurrencies”, "cryptocoin”, "cryptocoin", "crypto coins", "digital currency" and "virtual currency". The words "bitcoin" and "bitcoins" were also included due to the relevance of Bitcoin as the first cryptocurrency created.

The search resulted in 565 articles. An increase is perceived in the number of papers related to cryptocurrencies since Nakamoto's publication in 2008 - see Figure 3.

The search results were exported to Microsoft Excel tool, and two researchers reviewed the Title and Abstract (and the full text when needed) of each one of the 565 articles in order to select only the articles that have cryptocurrency as the main subject. Each researcher performed the categorization separately, and then both compared and discussed the differences found. Based on this first analysis, 162 articles were excluded because their purpose was not directly related to cryptocurrency, they have only cited cryptocurrency as an example for other subjects (62 articles), or their focus was 
Definition of study research questions:

a) what are the main topics presented in the literature on cryptocurrencies?

b) what are the main discussions and controversies on each topic presented in the literature?

Query in the Web of Science database:
Topic (Abstract, Title and Keywords): "bitcoin", "bitcoins", "crypto-currency", "crypto-
currencies", "cryptocurrency", "cryptocurrencies", "crypto currency", "crypto currencies",
"cryptocoin", "crypto-coin","cryptocoins", "digital currency" and "virtual currency"
Document Type: Articles type
Establishment of selection criteria of the articles found:
a) articles that has cryptocurrency as main subject
b) not related to the technology aspects of cryptocurrency (as blockchain)

Search in the database
565 articles found

Comparison of the two analyzes
a) Two researchers analyzed the Title, Abstract and (full text when needed) and categorize the
article according it main topic.
b) the results of both researchers where compared and the differences discussed

\begin{tabular}{|l|}
$\begin{array}{c}\text { Aplication of the selection and exclusion criteria } \\
\text { a) } 206 \text { articles excluded for lack of adherence to the review objective } \\
\text { b) } 359 \text { had the focus of the review }\end{array}$ \\
\hline $\begin{array}{c}\text { Critical analysis of the articles included in the analysis } \\
\text { Summary and conclusion of the evidences found }\end{array}$ \\
\hline
\end{tabular}

Figure 2. Design of the systematic literature review process

Source: developed by the authors based on Sampaio and Mancini (2007) 


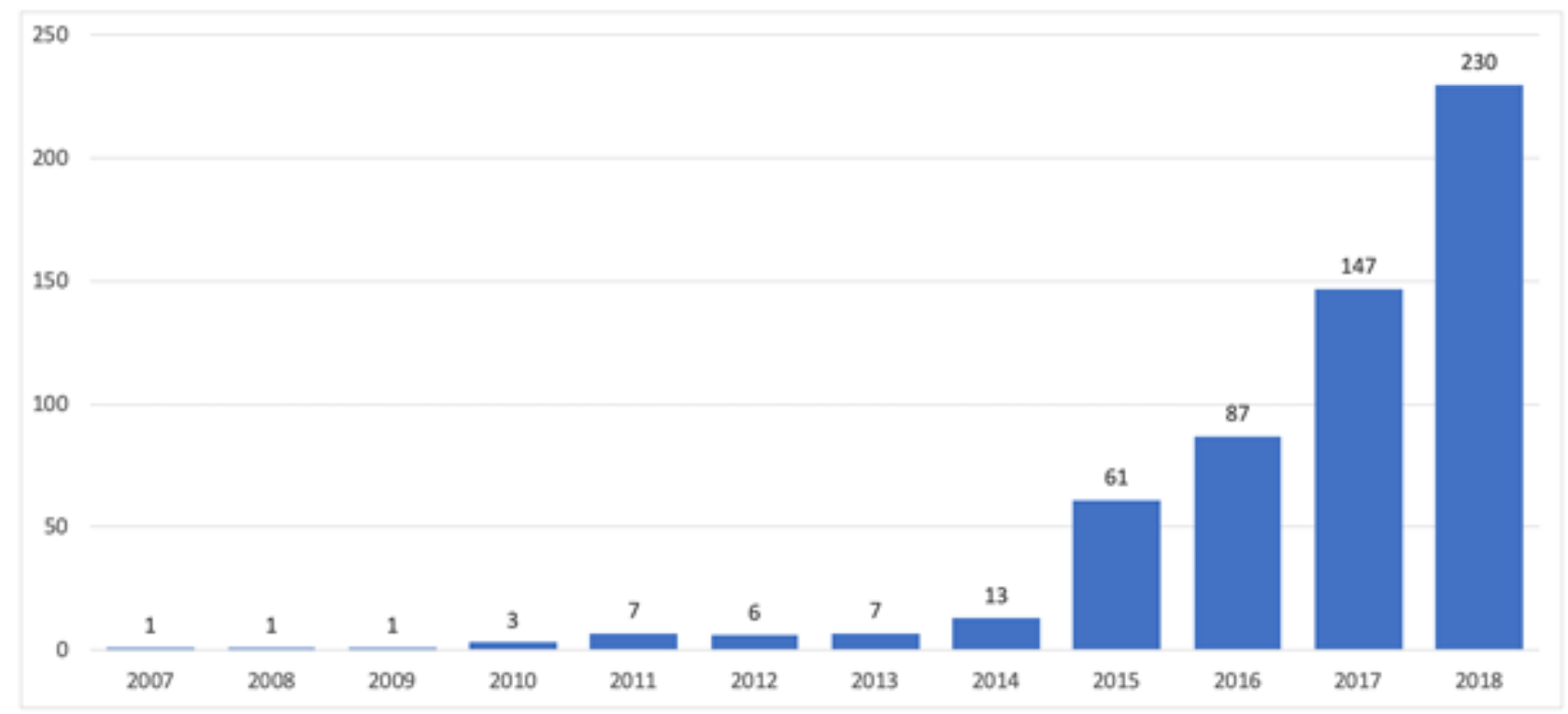

Figure 3. Cryptocurrency research by year

Source: Web of Science

on the blockchain technology (96 articles) or the full text was not available for further analysis (4 articles). Besides this, 44 articles discuss the technology (blockchain) with focus on cryptocurrency as enhancements for this technology and were also excluded.

The 359 remaining articles were downloaded and their content was categorized in the topics listed in the following section, that emerged during the analysis according to the purpose of the article.

\section{RESULTS}

In Chart 1 below, an overview of the state of art of cryptocurrencies studies is presented, as found in this review. The number of articles found per topic was also delimited.

The most frequent research topic (Figure 4, topic 1) is the economics of cryptocurrency (its exchange rate, price fluctuation, volatility) with 139 articles; 89 articles are devoted to the discussion of the nature, definition, and applications of cryptocurrency (topic 2), for instance: if it is it money or not, considering theories of money (Šurda, 2014; Bjerg, 2016). Law and regulation are also addressed (topic 3) as crimes related to cryptocurrency (topic 4) and the security, privacy and anonymity concerns (topic 5). Besides this, the future of this technology (topic 6) is also a key topic identified.

Together, 228 articles are related to the definition and economics of cryptocurrency (topics 1 and 2 in Figure 4). As explained by Fry and Cheah (2016, p. 345), "in the literature it remains unclear as to whether or not [...] cryptocurrencies should be seen as an alternative currency or as a speculative asset". Money can be defined in terms of three characteristics: a medium of exchange, store of value and unit of account (Bjerg, 2016; Smit, Buekens, \& Du Plessis, 2016; Bariviera, Basgall, Hasperue, $\&$ Naiouf, 2017). There is a consensus that crypto-coins are a means of exchange (a digital) by most authors, yet there is current research showing that more people are using the cryptocurrency for investment purposes than as a medium of exchange (Baur, Hong, \& Lee, 2018). However, there is no agreement about the store of value and unit of account. Smit et al. (2016) identified the three 
Chart 1. Key research topics

\begin{tabular}{|c|c|c|}
\hline Main topic & Main subjects & Number of articles \\
\hline Economic aspects, market and price & Exchange rates, price fluctuation, volatility & 139 \\
\hline $\begin{array}{l}\text { Discussion about cryptocurrencies definition } \\
\text { and applications; if they are }\end{array}$ & Money or not; theories of money; alternative currency or speculative asset & 89 \\
\hline Law and regulation for cryptocurrency & $\begin{array}{l}\text { Regulation as a factor to define cryptocurrencies; countries with } \\
\text { regulations versus countries without regulations; new laws and prohibition } \\
\text { of cryptocurrency use }\end{array}$ & 49 \\
\hline $\begin{array}{l}\text { Crimes involving cryptocurrency (through } \\
\text { cryptocurrency) }\end{array}$ & $\begin{array}{l}\text { Tax heaven; tax evasion; money laundering; business through the deep } \\
\text { web as drugs and terrorism }\end{array}$ & 30 \\
\hline Security, privacy and anonymity & $\begin{array}{l}\text { Cryptography; mining processes; solutions for the malleability of } \\
\text { transactions; anonymity; trust in the system }\end{array}$ & 30 \\
\hline Discussion about the future of cryptocurrencies & $\begin{array}{l}\text { Uncertainty; lack of information about the performance, reliability, and } \\
\text { cost of operating blockchains; earthcoin (future universal cryptocurrency); } \\
\text { market without future }\end{array}$ & 23 \\
\hline Total & & 359 \\
\hline
\end{tabular}

Source: the literature review

characteristics of money in the cryptocurrencies. Others, as Bariviera et al. (2017) concluded that cryptocurrencies cannot be considered money, mainly due to its instability, not attending the store of value function. Swartz (2018) said it is just igniting debates about the rule of money in the society, but it has not been used as money.

Cryptocurrencies, such as Bitcoin, Ethereum and Litecoin, are gaining attention due to this instability that is causing a high market price and has attracted investors (Bohme, Christin, Edelman, \& Moore, 2015; Zhu, Dickinson, \& Li, 2017b), but, at the same time, keeping them cautious, given the unclear conditions regarding market liquidity and security rules, generating vulnerability in transactions (Scaillet, Treccani, \& Trevisan, 2017). In one year (November 2016-2017), Bitcoin price has raised more than $871 \%$, Ethereum $2493 \%$ and Litecoin $1155 \%$ (Coinbase, 2017).

Many studies are trying to explain and predict these exchange rates. Kim (2015) argued that cryptocurrencies prices can be explained due to the decentralized control structure, the primary purpose of Nakamoto (2008). Other authors argued that higher exchange rates are caused by speculation, instead of cryptocurrencies being used as a medium of exchange (Cheah \& Fry, 2015; Zhu et al., 2017b). Li and Wang (2017) agree that speculation could explain the cryptocurrency market price, but only during the early market. Now, according to Denning and Lewis (2017), it is reacting to economic conditions. Bariviera et al. (2017) analyze the substantial volatility of Bitcoin and predict that its price will be reduced over time.

Currently, more than understanding the reasons behind the exchange rate, Kim et al. (2016) proposed a method to predict fluctuations in the prices of cryptocurrencies through the user comments and posted replies in online communities. Kristoufek (2013) compares internet searches (Google and Wikipedia), and Garcia, Tessone, Mavrodiev, and Perony (2014) analyzed Twitter and Internet (Google Trends) data to explain Bitcoin price changes, for example. However, Wang and Vergne (2017) argued that maybe cryptocurrency price variations cannot be explained by the analysis of online information or current money theories. They explain that cryptocurrency is a technology that consists of a real innovation potential and cannot be compared to traditional currencies or commodities. Li and Wang (2017) agreed with them and stated that the market lacks a proper understanding of the new technology and this drives the exchange rate in the early market. Bariviera et al. (2017), highlight the need for further research to understand the cryptocurrency dynamics across time. 
In another stream of research, studies review laws and regulations about cryptocurrency. According to Ishikawa (2017); (Prasad, Shankar, Gupta, \& Roy, 2018), the legal status of cryptocurrencies is still under debate. Governments are trying to accommodate the cryptocurrency under existing laws (European Central Bank, 2012; Ishikawa, 2017), creating new laws (Pieters \& Vivanco, 2017) or banning its use as occurred in Bangladesh, Bolivia, Ecuador, Iceland, Kyrgyzstan, and Vietnam (Gainsbury \& Blaszczynski, 2017; Hendrickson \& Luther, 2017; Zhu, Dickinson, \& Li, 2017a). In Japan, for instance, cryptocurrency is not defined as money, but as a proprietary value that can be exchanged (Ishikawa, 2017). In Brazil, cryptocurrencies have no definition yet, and so they cannot be submitted to the existing laws (Ribeiro, 2018).

In the absence of definitions, regulations and a central authority (as a bank), cryptocurrency has been considered as tax heaven, used for tax evasion and money laundering (Marian, 2013; Reid \& Harrigan, 2013; Bjerg, 2016). In addition to this, cryptocurrencies are being used to trade services and goods in the deep web (hidden Internet where illegal services and goods can be traded, like drugs (Aste et al., 2017) and for funding terrorists (Hendrickson, Hogan, \& Luther, 2016), due to their anonymity feature. In a traditional bank account system, if illegal activities are identified, accounts can be frozen, transactions can be reversed and account holders can be identified - that is not possible in cryptocurrency schemes (Hendrickson et al., 2016). Meiklejohn et al. (2013) explain that in these schemes the transactions are public to everyone, but the payer and payee are not identified. However, Reid and Harrigan (2013) demonstrate that it is possible to break down the anonymity and identify the users.

Besides anonymity, security is critical to trust a system to the point of exchanging fiat money for cryptocurrencies. The security of cryptocurrency is ensured by cryptography and mining processes (European Central European Central Bank, 2012; Redshaw, 2017). According to Nakamoto (2008) the incentive encourages nodes to stay honest. Even Nakamoto ensuring the security on cryptocurrency scheme based in the miner's honesty breaches due to security flaws allowed hackers to steal 350 million US dollars in 2014 from the Bitcoin wallet of Mt. Gox (Scott, Loonam, \& Kumar, 2017). So, security seems to be partially correct, especially when using digital wallets saved in a third part - where malicious attacks appear to be worth it. However, it is essential to highlight the efforts that have been made to overcome security flaws, such as proposed by Rajput, Abbas, and Oh (2018). They present, as an alternative, a robust mechanism capable of detecting any malleability of transactions that is an attack in which the attacker can alter or manipulate the transaction.

These discussions aim to understand the future of cryptocurrency. As Denning and Lewis (2017) stand, this future is not clear due to issues including performance, volatility, reliability and overall world energy cost of operating blockchains. Kewell and Ward (2017) concluded that there is a possibility of the emergence of a future universal cryptocurrency (earthcoin). However, new regulations and laws to be created can change this future (Kewell \& Ward, 2017). Luther (2016) does not see a favorable scenario for cryptocurrencies in the future, stating that they will probably be used for niche money or in countries with weak currencies. So, cryptocurrencies' future is surrounded by uncertainty. Therefore, regarding the literature, it is possible to conclude that cryptocurrency is a complex topic, involving the interaction of many different actors with different interests and understandings on this concept; it is important to understand the controversies they generate. The ANT is a theoretical lens that can be used to this purpose, as discussed next. 


\section{ACTOR-NETWORK THEORY (ANT)}

ANT is a social theory that examines the relationship, motivations, and actions between the actors of a network (Walsham, 1997; Twum-Darko \& Harker, 2017). These actors can be human or non-human entities, including technologies, elements of nature, pieces of art, etc. that play roles make a difference or act in the network (Latour, 2005; Venturini \& Latour, 2010). According to Hanseth, Aanestad, and Berg (2004), all networks are composed of humans and technological components. ANT is concerned with mapping the actions and roles of these actors in the network (Elbanna, 2012).

According to Elbanna (2012), a network is built to achieve a goal and, through a translation process, the "network builder recruits actors and ensures their faithful alliance". Translation is a core concept of ANT (Callon, 1984; Latour, 2005) and can be defined as the alignment process of the actors in a network with the network builder (Ahmedshareef, Hughes, \& Petridis, 2014; TwumDarko \& Harker, 2017) During the translation, focal actors frame a problem, analyze possible solutions (problematisation) and define one of them as the obligatory passage point (OPP) from all the other actors in the network (Twum-Darko \& Harker, 2017). Then, the focal actors start a process of negotiation with the actors in order to maintain the interests aligned (interessement), define their roles in the network (enrollment) and assume a role of representatives of the network, mobilizing the actors into action (mobilization) (Shim \& Shin, 2016; Twum-Darko \& Harker, 2017).

In summary, focal actors mobilize, negotiate and persuade other actors to act for achieving a specific goal. These actions can determine the success or failure of the network (Bonner, 2013). When the outcomes of the network become irreversible and taken for granted, black boxes emerge (Bonner, 2013). However, as stated by Hanseth et al. (2004, p. 119), the "central idea and motivation behind ANT is to study the construction of things normally taken for granted". New controversies can emerge from black boxes. They are situations in which the actors disagree (Venturini, 2010b) and to close them, a consensus among them should emerge (Law \& Bijker, 1992; Venturini \& Latour, 2010). Venturini (2010a, p. 17), said the "controversies are the most dynamic phenomena of collective life" and "they need to be explored in time". In addition, Callon, Lascoumes, and Barthe (2009) explain that controversies reveal uncertainties and new lines of research can be explored.

\section{EXPLORING CONTROVERSIES ON CRYPTOCURRENCIES}

The cartography of controversies is a method developed by ANT authors to train students, researchers and journalists to investigate contemporary socio-technical issues; it is an exercise of crafting devices to observe and describe the controversies (Venturini, 2010b) Also, tracking the controversies is beneficial for observing and understanding innovations such as cryptocurrencies. Through the literature review on this subject - cryptocurrencies - it is possible to observe that there are common issues under debate and almost no consensus around them. Venturini (2010b) presents some key features of social controversies that can be considered to analyze the debate on cryptocurrencies.

\section{CONTROVERSIES INVOLVE MANY DIFFERENT ACTORS}

Cryptocurrency controversies involve humans and non-human actors, starting with Nakamoto (whose real identity is still unknown), developers, "hippies, anarchists, cyberpunks, cryptographers, payment systems experts, currency activists, commodity traders" (Maurer et al., 2013) "miners", 
speculators, investors, drug dealers (Van Hout \& Bingham, 2013), institutions, banks, government (from several countries), police, FBI (Meiklejohn et al., 2013), the economy, technology (blockchain), computers around the world, the deep web, current money as it is known, electricity, and even some curious people. Different actors, different interests, different motivations, roles, and goals. Many networks, many black boxes to be closed. Some actors create networks for maintaining the cryptocurrency system for different purposes ("miners", investors) and others for the opposite (e.g. banks). Some people want to use the cryptocurrency system to avoid paying fees to a bank and challenge the current payment systems, while others use it as a means to criminal operations such as selling drugs.

\section{CONTROVERSIES UNVEIL THE SOCIAL IN ITS MOST DYNAMIC FORM}

Some years ago, we could not have imagined that the definition of money would be in such an intense debate today. This change can affect the collective life in many forms if everyone can create its own money (its own cryptocurrency). Besides this, without a central authority in the cryptocurrency scheme, what will happen with the banks that control the money and transactions nowadays? Will they disappear, or will they play new roles in the network?

Even if there is a technical explanation about the cryptocurrency system, it is not clear, for nontechnical people, where the cryptocurrency value is stored or how it is controlled, mainly because it is distributed around the world in thousands of computers. If something happens in this network, what will happen with the cryptocurrency and the values invested in this new kind of "money"? Alternatively, what/who is controlling this technology? Do someone or something control it?

As stated by Maurer et al. (2013), the novelty about cryptocurrency is not related to cryptography, anonymity, economics or the nature of money. More than that, people trust the code itself(blockchain), "substituting the credibility of persons, institutions, and governments" (Maurer et al., 2013, p. 263). This change can provide a useful reflection about the social, about how imbricated are the human and non-human elements in our society, in which many people do not trust others nor social institutions, but trust codes and software (developed and maintained by humans). How to explain this complex and dynamic scenario? Moreover, when some actors do not agree with some features of a cryptocurrency, new cryptocurrencies could be created (Low \& Teo, 2017), following new rules.

\section{CONTROVERSIES ARE DEBATED AND REDUCTION-RESISTANT}

Cryptocurrency was released in 2009, but a consistent increase in the researches about this topic can be seen only in 2014 - what happened in this meantime? Our study was based on the scientific literature, but accessing the Internet (Forbes, The Economist, Twitter, etc.), it is possible to realize that controversies around cryptocurrency are far from being closed.

Do actors disagree in almost everything related to cryptocurrencies: Is it money or not? Is it a trend or not? Will cryptocurrency replace the money as we know it or not? Will there be some institution controlling the transactions? Will the anonymity be kept? Will cryptocurrency be banned? Will it be used for illegal activities? How will the police track these activities? Will the cryptocurrency market be stable? Which effects will cryptocurrencies leave in the economy? The debate is ongoing and surrounded by uncertainties. More than that, there are not only one or three cryptocurrencies: new ones have been created almost every day. As (Venturini, 2010b, p. 262) states, "the difficulty 
of controversy is not that actors disagree on answers, but that they cannot even agree on questions". Probably, new questions will arise, maybe closing some debates, but creating new ones and do not letting the black boxes to be closed so soon.

Before cryptocurrency, money, currencies, banks, payment systems and economic systems were taken for granted, even if new ways of payment emerged (as mobile payments). As previously presented, economists and theorists are questioning what is "real" money or not, reviewing the theories of money, market price and volatility. People are questioning the need of banks, the need of central authorities on payment systems, the need of fees and the need of governments imposing laws on money and even why current coins are needed instead of a global currency, as the earthcoin proposed by Kewell and Ward (2017).

\section{RESEARCH TOPICS ON CRYPTOCURRENCY CONTROVERSIES}

We can conclude that a mine of controversies can be found about Cryptocurrency and need to be explored. Considering the previous literature review and some essential procedures to map controversies indicated by Venturini (2010b), we suggest a list of research topics on this subject.

- Understanding the non-controversial elements: basic concepts about the technology involved in Cryptocurrency could be explored and analyzed in order to reduce the confuse understanding of how the cryptocurrency systems work and what they can imply.

- Analyzing the "tree of disagreement": Venturini (2010b, p. 260) said, "the construction of a shared universe is often accompanied by the clash of conflicting worlds". Analyzing the actors previously mentioned, the conflicts are clear: police and drug dealers; government and tax evaders; banks and cryptocurrency systems; economists and speculators. (Venturini, 2010b, p. 26) claim that "controversies decide and are decided by the distribution of power". Could the governments or the police be more powerful than a distributed technology with thousands of computers around the world? The disagreements and power struggles among the actors involved in the cryptocurrency debate deserve further analysis.

- Analyzing the "scale of the controversy": no controversy is isolated; they are usually related to other controversies (Venturini, 2010b). It is the case of the Cryptocurrency, which can be related to other controversies such as those that question the power of banks and central governments, hacker movements, privacy debates, and regulations. Therefore, another way of disentangling the complexity of cryptocurrencies is analyzing the other controversies that surround it.

- Tracing the "diagram of actor-networks": according to Venturini (2010a, p. 806) "every actor can be decomposed into a network and that every network can be connected tightly enough to become a single actor", so more research is needed to trace the connections between the human and non-human actors involved with cryptocurrencies systems and how they become increasingly intertwined.

- Analyzing the cosmoses: controversies involve the opposition of conflicting worldviews. Future research can analyze the different and multiple discourses from different actors involved with cryptocurrencies, for the present and expectations for the future, according to the different actor's views. 
These are just starting points to study the controversies on cryptocurrency. It is essential to identify the focal actors and their goals in each network, the other actors involved and the roles they play.

\section{FINAL REMARKS}

Cryptocurrency is a topic surrounded by controversies. Considering that this topic is attracting the attention of many disciplines, the purpose of this article was to present an overview about cryptocurrency studies, arguing that it is possible to be analyzed as a controversy through the lens of the Actor-Network Theory. Cryptocurrency is a remarkable innovation, but its complexity and the lack of understanding about it is generating many debates and conflicts between several actors around the world, each one with specific interests in using this technology. Cryptocurrency has opened several black boxes regarding the payment systems and the concept of money itself, and maybe it is creating new concepts. A "mine" full of controversies can be explored in this context.

The purpose of this article was not to map the controversies but to demonstrate that, through the ANT and the method of controversy mapping (Venturini, 2010b), there is a possibility to answer some of the questions raised about cryptocurrencies and, especially, its future developments. Taking this into account, future research can be conducted to map the controversies related to this topic providing some answers about the future developments of complex cryptocurrency systems. As limitations, this article is based in the literature available in the Web of Science database. During our search, proceedings papers demonstrated to have potential to present different points of views (new actors or new controversies) and, for future research these works, as well as Internet data (forums, communities, non-academic journals), need also to be considered.

\section{REFERENCES}

Ahmedshareef, Z., Hughes, R., \& Petridis, M. (2014). Exposing the influencing factors on software project delay with actor-network theory. Electronic Journal of Business Research Methods, 12(2), 132-146.

Aste, T., Tasca, P., \& Di Matteo, T. (2017). Blockchain Technologies: The Foreseeable Impact on Society and Industry. Computer, 50(9), 18-28. doi:10.1109/mc.2017.3571064

Barber, S., Boyen, X., Shi, E., \& Uzun, E. (2012). Bitter to better-how to make bitcoin a better currency. Paper presented at the International Conference on Financial Cryptography and Data Security.

Bariviera, A. F., Basgall, M. J., Hasperue, W., \& Naiouf, M. (2017). Some stylized facts of the Bitcoin market. Physica a-Statistical Mechanics and Its Applications, 484, 82-90. doi:10.1016/j. physa.2017.04.159

Bariviera, A. F., Zunino, L., \& Rosso, O. A. (2018). An analysis of high-frequency cryptocurrencies prices dynamics using permutation-information-theory quantifiers. Chaos, 28(7), 7. doi:10.1063/1.5027153

Baur, D. G., Hong, K., \& Lee, A. D. (2018). Bitcoin: Medium of exchange or speculative assets? Journal of International Financial Markets Institutions \& Money, 54, 177-189. doi:10.1016/j.intfin.2017.12.004

Bjerg, O. (2016). How is Bitcoin Money? Theory Culture \& Society, 33(1), 53-72. doi:10.1177/0263276415619015

Bohme, R., Christin, N., Edelman, B., \& Moore, T. (2015). Bitcoin: Economics, Technology, and Governance. Journal of Economic Perspectives, 29(2), 213-238. doi:10.1257/jep.29.2.213 
Bonner, W. B. (2013). History and IS-Broadening our view and understanding: Actor-Network Theory as a methodology. Journal of information technology, 28(2), 111-123.

Callon, M. (1984). Some elements of a sociology of translation: domestication of the scallops and the fishermen of St Brieuc Bay. The Sociological Review, 32(1_suppl), 196-233.

Callon, M., Lascoumes, P., \& Barthe, Y. (2009). Acting in an Uncertain World: An Essay on Technical Democracy: The MIT Press.

Cheah, E. T., \& Fry, J. (2015). Speculative bubbles in Bitcoin markets? An empirical investigation into the fundamental value of Bitcoin. Economics Letters, 130, 32-36. doi:10.1016/j.econlet.2015.02.029

Coinbase. (2017). Graphics. Retrieved November 2, 2017 from https://coinbase.com

Denning, P. J., \& Lewis, T. G. (2017). Bitcoins Maybe; Blockchains Likely. American Scientist, 105(6), $335-$ 339.

Elbanna, A. (2012). Applying Actor Network Theory and Managing Controversy. In W. M. Dwivedi Y., Schneberger S (Ed.), Information Systems Theory. Integrated Series in Information Systems (Vol. 29, pp. 117-129). New York, NY: Springer.

European Central Bank. (2012). Virtual currency schemes. Retrieved October 20, 2017 from https://www. ecb.europa.eu/pub/pdf/other/virtualcurrencyschemes201210en.pdf

Fry, J., \& Cheah, E. T. (2016). Negative bubbles and shocks in cryptocurrency markets. International Review of Financial Analysis, 47, 343-352. doi:10.1016/j.irfa.2016.02.008

Gainsbury, S. M., \& Blaszczynski, A. (2017). How Blockchain and cryptocurrency technology could revolutionize online gambling. Gaming Law Review-Economics Regulation Compliance and Policy, 21(7), 482-492. doi:10.1089/glr2.2017.2174

Garcia, D., Tessone, C. J., Mavrodiev, P., \& Perony, N. (2014). The digital traces of bubbles: feedback cycles between socio-economic signals in the Bitcoin economy. Journal of the Royal Society Interface, 11(99), 8. doi:10.1098/rsif.2014.0623

Hanseth, O., Aanestad, M., \& Berg, M. (2004). Guest editors' introduction: Actor-network theory and information systems. What's so special? Information Technology \& People, 17(2), 116-123.

Hendrickson, J. R., Hogan, T. L., \& Luther, W. J. (2016). The political economy of Bitcoin. Economic Inquiry, 54(2), 925-939. doi:10.1111/ecin.12291

Hendrickson, J. R., \& Luther, W. J. (2017). Banning bitcoin. Journal of Economic Behavior \& Organization, 141, 188-195. doi:10.1016/j.jebo.2017.07.001

Ishikawa, M. (2017). Designing Virtual Currency Regulation in Japan: Lessons from the Mt Gox Case. Journal of Financial Regulation, 3(1), 125-131. doi:10.1093/jfr/fjw015

Kewell, B., \& Ward, P. M. (2017). Blockchain futures: With or without Bitcoin? Strategic Change-Briefings in Entrepreneurial Finance, 26(5), 491-498. doi:10.1002/jsc.2149

Kim, T. (2015). The Predecessors of Bitcoin and Their Implications for the Prospect of Virtual Currencies. Plos One, 10(4), 18. doi:10.1371/journal.pone.0123071

Kim, Y. B., Kim, J. G., Kim, W., Im, J. H., Kim, T. H., Kang, S. J., \& Kim, C. H. (2016). Predicting Fluctuations in Cryptocurrency Transactions Based on User Comments and Replies. Plos One, 11(8), 17. doi:10.1371/journal.pone.0161197

Kristoufek, L. (2013). BitCoin meets Google Trends and Wikipedia: Quantifying the relationship between phenomena of the Internet era. Scientific Reports, 3, 7. doi:10.1038/srep03415 
Latour, B. (2005). Reassembling the social: An introduction to actor-network-theory: Oxford university press.

Law, J., \& Bijker, W. E. (1992). Postscript: Technology, stability and social theory. WE Bijker \& J. Law (Eds.), Shaping technology/building society: Studies in sociotechnical change, 290-308.

Li, X., \& Wang, C. A. (2017). The technology and economic determinants of cryptocurrency exchange rates: The case of Bitcoin. Decision Support Systems, 95, 49-60. doi:10.1016/j.dss.2016.12.001

Low, K. F., \& Teo, E. G. (2017). Bitcoins and other cryptocurrencies as property? Law, Innovation and Technology, 9(2), 235-268.

Luther, W., \& White, L. (2014). Can bitcoin become a major currency?

Luther, W. J. (2016). Bitcoin and the Future of Digital Payments. Independent Review, 20(3), 397-404.

Marian, O. (2013). Are Cryptocurrencies Super Tax Havens? Michigan Law Review First Impressions, 38, 112.

Maurer, B., Nelms, T. C., \& Swartz, L. (2013). "When perhaps the real problem is money itself!": the practical materiality of Bitcoin. Social Semiotics, 23(2), 261-277. doi:10.1080/10350330.2013.777594

Meiklejohn, S., Pomarole, M., Jordan, G., Levchenko, K., McCoy, D., Voelker, G. M., \& Savage, S. (2013). A fistful of bitcoins: characterizing payments among men with no names. Paper presented at the Proceedings of the 2013 conference on Internet measurement conference.

Moore, T. (2013). The promise and perils of digital currencies. International Journal of Critical Infrastructure Protection, 6(3-4), 147-149. doi:10.1016/j.ijcip.2013.08.002

Nakamoto, S. (2008). Bitcoin: A peer-to-peer electronic cash system. Retrieved October 10, 2017 from https://bitcoin.org/bitcoin.pdf

Osterrieder, J., \& Lorenz, J. (2017). A STATISTICAL RISK ASSESSMENT OF BITCOIN AND ITS EXTREME TAIL BEHAVIOR. Annals of Financial Economics, 12(1), 19. doi:10.1142/ s2010495217500038

Pieters, G., \& Vivanco, S. (2017). Financial regulations and price inconsistencies across Bitcoin markets. Information Economics and Policy, 39, 1-14. doi:10.1016/j.infoecopol.2017.02.002

Prasad, S., Shankar, R., Gupta, R., \& Roy, S. (2018). A TISM modeling of critical success factors of blockchain based cloud services. Journal of Advances in Management Research, 15(4), 434-456. doi:10.1108/jamr-03-2018-0027

Rahman, A. J. (2018). Deflationary policy under digital and fiat currency competition. Research in Economics, 72(2), 171-180. doi:10.1016/j.rie.2018.04.004

Rajput, U., Abbas, F., \& Oh, H. (2018). A Solution towards Eliminating Transaction Malleability in Bitcoin. Journal of Information Processing Systems, 14(4), 837-850. doi:10.3745/jips.03.0101

Redshaw, T. (2017). Bitcoin beyond ambivalence: Popular rationalization and Feenberg's technical politics. Thesis Eleven, 138(1), 46-64. doi:10.1177/0725513616689390

Reid, F., \& Harrigan, M. (2013). An analysis of anonymity in the bitcoin system. In Security and privacy in social networks (pp. 197-223): Springer.

Ribeiro, R. M. L. (2018). Bitcoin in the Brazilian Financial System. Revista Tecnologia E Sociedade, 14(33), 16.

Sampaio, R. F., \& Mancini, M. C. (2007). Estudos de revisão sistemática: um guia para síntese criteriosa da evidência científica. Revista brasileira de fisioterapia, 11(1), 83-89. 
Scaillet, O., Treccani, A., \& Trevisan, C. (2017). High-frequency jump analysis of the bitcoin market.

Scott, B., Loonam, J., \& Kumar, V. (2017). Exploring the rise of blockchain technology: Towards distributed collaborative organizations. Strategic Change-Briefings in Entrepreneurial Finance, 26(5), 423-428. doi:10.1002/jsc. 2142

Shim, Y., \& Shin, D.-H. (2016). Analyzing China's fintech industry from the perspective of actor-network theory. Telecommunications Policy, 40(2-3), 168-181.

Smit, J. P., Buekens, F., \& Du Plessis, S. (2016). Cigarettes, dollars and bitcoins - an essay on the ontology of money. Journal of Institutional Economics, 12(2), 327-347. doi:10.1017/s1744137415000405

Šurda, P. (2014). The origin, classification and utility of Bitcoin.

Swartz, L. (2018). What was Bitcoin, what will it be? The techno-economic imaginaries of a new money technology. Cultural Studies, 32(4), 623-650. doi:10.1080/09502386.2017.1416420

Tschorsch, F., \& Scheuermann, B. (2016). Bitcoin and Beyond: A Technical Survey on Decentralized Digital Currencies. Ieee Communications Surveys and Tutorials, 18(3), 2084-2123. doi:10.1109/ comst.2016.2535718

Twum-Darko, M., \& Harker, L.-A. L. (2017). Understanding Knowledge Sharing in an Organization: A Perspective of Actor-Network Theory. International Journal of Knowledge Management (IJKM), 13(1), 53-74.

Urquhart, A. (2016). The inefficiency of Bitcoin. Economics Letters, 148, 80-82. doi:10.1016/j. econlet.2016.09.019

Van Hout, M. C., \& Bingham, T. (2013). 'Surfing the Silk Road': A study of users' experiences. International Journal of Drug Policy, 24(6), 524-529. doi:10.1016/j.drugpo.2013.08.011

Venturini, T. (2010a). Building on faults: how to represent controversies with digital methods. Public understanding of science, 21(7), 796-812.

Venturini, T. (2010b). Diving in magma: how to explore controversies with actor-network theory. Public understanding of science, 19(3), 258-273.

Venturini, T., \& Latour, B. (2010). The Social Fabric: Digital Footprints and Qualiquantitative Methods. Proceedings of futur en Seine, 87-101.

Walsham, G. (1997). Actor-network theory and IS research: current status and future prospects. In Information systems and qualitative research (pp. 466-480): Springer.

Wang, S., \& Vergne, J. P. (2017). Buzz Factor or Innovation Potential: What Explains Cryptocurrencies' Returns? Plos One, 12(5), 1. doi:10.1371/journal.pone.0177659

Webster, J., \& Watson, R. T. (2002). Analyzing the past to prepare for the future: Writing a literature review. MIS quarterly, xiii-xxiii.

World Economic Forum. (2016). All you need to know about blockchain, explained simply. Retrieved November 1, 2017 from https://goo.gl/bRLQhN

Zhu, Y. C., Dickinson, D., \& Li, J. J. (2017a). Analysis on the influence factors of Bitcoin's price based on VEC model. Financial Innovation, 3(1), 13. doi:10.1186/s40854-017-0054-0

Zhu, Y. C., Dickinson, D., \& Li, J. J. (2017b). Analysis on the influence factors of Bitcoin's price based on VEC model (vol 3, 3, 2017). Financial Innovation, 3(1), 1. doi:10.1186/s40854-017-0057-x

Zimmer, Z. (2017). Bitcoin and Potosi Silver Historical Perspectives on Cryptocurrency. Technology and Culture, 58(2), 307-334. doi:10.1353/tech.2017.0038 